\title{
APLICATIVO PARA APOIO AO PROCESSO ENSINO-APRENDIZAGEM DE CÁLCULO E DILUIÇÃO DE MEDICAMENTOS E DE GOTEJAMENTO DE SORO
}

\author{
RIBEIRÃO PRETO/SP JULHO/2018
}

\author{
Lucas da Cunha Alves - UNAERP - Icalves012@gmail.com \\ Gabriel Bocato Ferreira \\ Alex Di Vennet Xicatto \\ - UNAERP - bielbocato@hotmail.com \\ - UNAERP - alexxicatto@gmail.com \\ Gabriela Barbosa Pegoraro \\ - UNAERP - pegoraro.gabriela@outlook.com \\ Silvia Sidnéia da Silva \\ - UNAERP - sssilva@unaerp.br \\ Edilson Carlos Caritá \\ - UNAERP - ecarita@unaerp.br
}

\author{
Tipo: Investigação Científica (IC) \\ Natureza: Relatório Final de Pesquisa \\ Categoria: Métodos e Tecnologias \\ Setor Educacional: EDUCAÇÃO SUPERIOR
}

\begin{abstract}
RESUMO
O objetivo do estudo é descrever o desenvolvimento de um aplicativo para apoiar o processo ensinoaprendizagem de estudantes de graduação em enfermagem referente ao cálculo e diluição de medicamentos, e gotejamento de soro. Trata-se de um estudo exploratório-descritivo com abordagem qualitativa realizada por meio de grupo focal. O app, denominado Dosage Helper, foi desenvolvido com o Android Studio versão 3.0.1 e o IDE IntelliJ IDEA. A linguagem Java foi usada para a implementação das interfaces e equações para simulação dos cálculos e diluição de medicamentos. $O$ aplicativo permite ao estudante de enfermagem interpretar as prescrições médicas, calcular a diluição de medicamentos, montar a equação (regra de três), visualizar texto explicativo de como o cálculo foi realizado e o resultado do cálculo. Objeto de Aprendizagem $(O A)$ foi validado por meio de uma análise qualitativa com um grupo focal constituído por duas docentes especialistas em farmacologia de uma Instituição de Ensino Superior privada do interior paulista. As docentes utilizaram o app, simularam diversos exemplos aplicados nas aulas e concluíram que as interfaces são amigáveis, o tempo de retorno das informações é adequado e os cálculos de diluição de medicamentos foram realizados corretamente, portanto, não recomendaram alterações. Desse modo, o OA pode ser disponibilizado como recurso didático-pedagógico para os alunos de graduação em enfermagem. Infere-se ainda que o app poderá ser oferecido como OA para apoio nas aulas de cursos presenciais, na modalidade Educação a Distância (EAD) ou híbrido (semipresenciais), pois permite aos alunos aplicarem a teoria, além de simular a prática, sendo mais uma estratégia didático-pedagógica inerente aos anseios das gerações contemporâneas que ocupam os espaços das escolas de educação superior, uma vez que as estimulam na continuidade da simulação do processo de cálculo e diluição de medicamentos, bem como do gotejamento de soro, intra e extra sala de aula.
\end{abstract}

Palavras-chave: Objeto de Aprendizagem. Enfermagem. Cálculo e Diluição de Medicamentos. Gotejamento de Soro. App. 


\section{INTRODUÇÃo}

No Brasil, a administração de medicamentos é atividade cotidiana e de responsabilidade legal da equipe de enfermagem, em todas as instituições de saúde e, portanto, revestese de grande importância tanto para essa categoria profissional quanto para os pacientes. Nessa direção, são necessárias equipes qualificadas e treinadas, a fim de evitar riscos ao paciente durante o processo de terapia medicamentosa.

Considerando o advento de inúmeros medicamentos no mercado e o avanço tecnológico da indústria farmacêutica, a administração de medicamentos tornou-se uma tarefa extremamente complexa, requerendo dos profissionais da saúde cada vez mais responsabilidades, pautando-se em conhecimentos relativos à farmacologia, anatomia e fisiologia, bem como habilidades técnicas.

A administração de medicação é ato constituído por vários processos como prescrição, revisão e validação da prescrição, distribuição ou dispensação, preparo, administração, e acompanhamento do paciente para monitoramento da ação ou reação ao medicamento. Entretanto, evidencia-se, muitas vezes, o despreparo da equipe de enfermagem em relação ao preparo e administração de medicamentos, o que resulta em erros relativos à medicação.

Os erros durante a preparação e administração de medicamentos representam uma dura realidade para os pacientes, profissionais de saúde e instituições hospitalares, acarretando sérias consequências a todos os envolvidos (CARVALHO, 2000).

Bates et al. (1999) afirmam que, em cada 100 pacientes admitidos nos hospitais, 4,8\% apresentam eventos adversos e os erros com a medicação ocorrem mais frequentemente nos estágios da prescrição médica (56\%), transcrição da prescrição médica $(6 \%)$, distribuição do medicamento (4\%) e administração do medicamento (34\%). Os erros relacionados à utilização de medicamentos podem resultar em sérias consequências para o paciente e sua família, como gerar incapacidades, prolongar o tempo de internação e de recuperação, expor o paciente a maior número de procedimentos e medidas terapêuticas, atrasar ou impedir que reassumam suas funções sociais, e até mesmo acarretando a morte.

Como constata-se na vivência profissional, o Conselho Regional de Enfermagem (COREn, 2011) ratifica que conhecer e aplicar adequadamente os fundamentos da aritmética e da matemática respalda o profissional de saúde quanto à prevenção de erros relacionados ao preparo, a dosagem e/ou à administração de medicamentos?. 
Nesse sentido, capacitar o profissional de enfermagem para realizar as operações matemáticas envolvidas nessa ação é atribuição do enfermeiro que, além de responder pela formação de auxiliares e técnicos de enfermagem, também gradua o enfermeiro que é o líder da equipe e se responsabiliza por revisar as dosagens prescritas, entre outros aspectos, nesse processo.

Para apoiar os processos de ensino-aprendizagem que envolvem simulação e/ou raciocínio lógico, contemporaneamente, pode-se usar a Tecnologia da Informação e Comunicação (TIC), especificamente Objetos de Aprendizagem (OA). Segundo Blanc e Benlloch-Dualde (2014), OA podem ser definidos como instrumentos digitais desenvolvidos para serem usados em unidades de ensino-aprendizagem, de forma independente e reutilizável. Para McGreal (2004), OA permite e facilita o uso de conteúdos educacionais interoperáveis e reutilizáveis em diversos ambientes de ensinoaprendizagem, constituindo-se em recursos educacionais que podem ser usados e reutilizados, em contextos distintos, para apoiar nesse processo.

Bistane et al. (2014) inferem que o uso de OA no processo ensino-aprendizagem com estudantes da área da saúde é um recurso didático-pedagógico que estimula a aquisição de conhecimentos, pois o torna dinâmico e atrativo e sua apresentação como app permite dialogar ativamente com as atuais gerações contemporâneas, presentes nas universidades.

\section{OBJETIVO}

O objetivo do estudo é descrever o desenvolvimento de um aplicativo para apoiar o processo ensino-aprendizagem de estudantes de graduação em enfermagem referente ao cálculo e diluição de medicamentos, e gotejamento de soro.

\section{MATERIAL E MÉTODOS}

Trata-se de um estudo exploratório-descritivo, com abordagem qualitativa, realizado por meio de grupo focal.

A ferramenta usada para o desenvolvimento do app foi o Android Studio versão 3.0.1 com o Integrated Development Environment (IDE) IntelliJ IDEA. A linguagem de programação Java foi usada para a implementação das interfaces e equações para simulação dos cálculos de diluição de medicamentos.

O aplicativo foi denominado Dosage Helper e permite ao estudante de enfermagem 
interpretar as prescrições médicas, calcular a diluição de medicamentos, montar a equação (regra de três), visualizar texto explicativo de como o cálculo foi realizado e o resultado do cálculo.

Para validação do app foi realizada análise qualitativa com um grupo focal constituído por duas docentes especialistas em farmacologia de uma Instituição de Ensino Superior privada do interior paulista. O grupo focal é uma técnica de entrevistas com grupos, baseada na comunicação e na interação, refere-se a uma discussão aberta sobre um contexto de interesse comum ao pesquisador e aos participantes, permitindo coletar relatos sobre a compreensão e as percepções de um tema, produto ou serviços (KITZINGER, 2000; GASKELL, 2002).

\section{RESULTADOS}

O aplicativo Dosage Helper possui sete interfaces, sendo a primeira a tela inicial (Figura 1), onde os estudantes podem selecionam a opção entrar ou obter instruções sobre o uso do aplicativo. Quando o usuário seleciona a opção entrar abre-se a segunda tela (Figura 2), que é o menu principal do app e contém a opção cálculo e diluição de medicamentos, e de gotejamento de soro.

Ao clicar na opção "cálculo e diluição de medicamentos" abre-se a terceira tela (Figura 3), e nela há a interface que o estudante utilizará para realizar o cálculo de diluição de medicamentos. O estudante poderá habilitar somente um " $X$ " no momento da simulação do cálculo de diluição de medicamentos e para obter o resultado, deverá preencher todos os campos com os números desejados e escolher a unidade de medida. O solvente 1 refere-se à quantidade de uma substância que deverá ser utilizada para a diluição; o soluto 1 é a quantidade de remédio; o solvente 2 é o total que se tem de remédio (disponível para uso) e, por fim, o soluto 2 é a quantidade existente de remédio. As unidades de medidas que os estudantes podem usar são: Litros (L), Mililitros (MI), Microgramas (Mcg); Unidades Internacionais (UI), Gramas (G), Gotas (Gt), Miligramas (Mg) e Microgotas (Mgt).

A quarta interface é responsável por apresentar o resultado da simulação de cálculo de diluição de medicamento (Figura 4). Ela contempla o resultado do cálculo de diluição de medicamentos e um texto explicativo de como este foi realizado. 

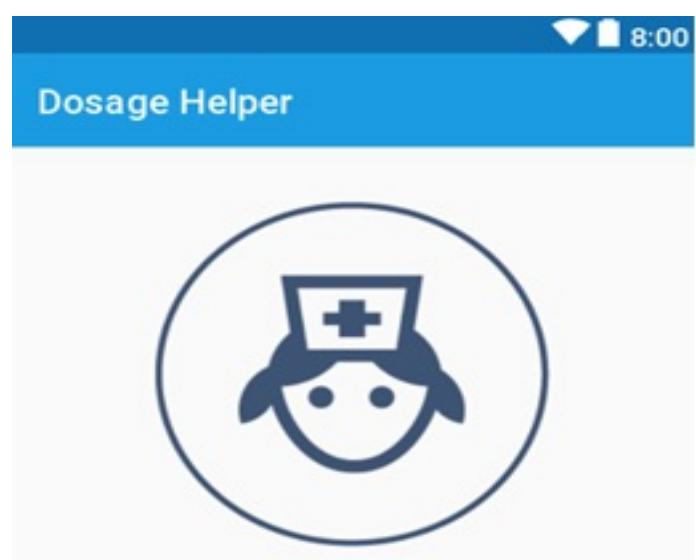

\section{ENTRAR}

\section{INSTRUÇŌES}

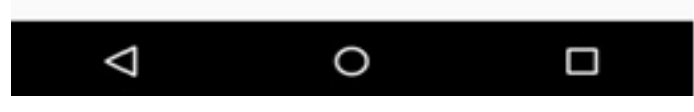

Figura 1 - Tela Inicial

0
Dosage Helper

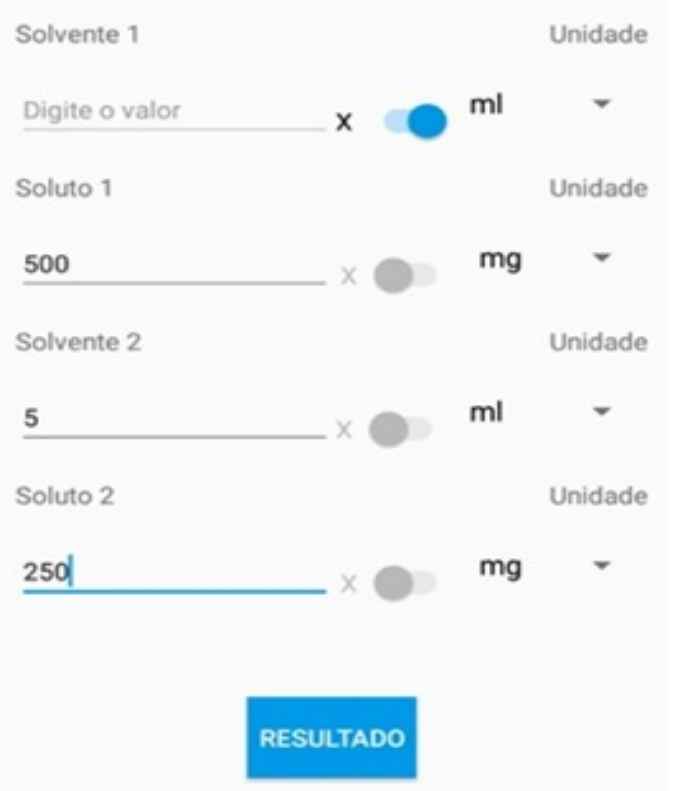

Figura 3 - Demonstração do cálculo e diluição de medicamentos

\section{CÁLCULO DE GOTEJAMENTO}

CÁLCULO E DILUIÇĀO DE MEDICAMENTOS

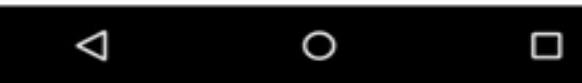

Figura 2 - Menu Principal

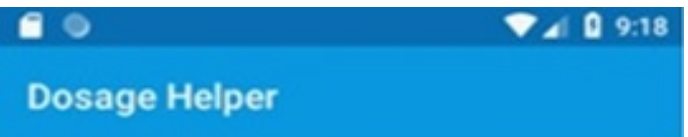

Em uma solução que contém $250.0 \mathrm{mg}$ em $5.0 \mathrm{ml}$, temos que diluir $500.0 \mathrm{mg}$ em $10.0 \mathrm{ml}$.

\section{Resultado: $10.0 \mathrm{ml}$}

Figura 4 - Resultado da simulação 
A quinta tela contempla o cálculo de gotejamento, e para simular esse cálculo, o estudante deverá informar a quantidade de medicamento que se deseja administrar e o tempo (em horas ou minutos) (Figura 5). O cálculo é feito a partir de duas equações, sendo número de gotas $/$ minuto $=(\mathrm{V} \times 20) /$ número de minutos, para o resultado em gotas $/$ minuto e número de gotas $/$ minuto $=\mathrm{V} /(\mathrm{T} \times 3)$, para o resultado em horas. $\mathrm{A}$ variável $\mathrm{V}$ refere-se ao volume em $\mathrm{ml}$ e a variável $\mathrm{T}$ ao tempo, em horas. Após o preenchimento dos campos, o estudante deve clicar em resultado e, na sequência, abrirá a sexta tela (Figura 6), que apresentará os resultados do cálculo.

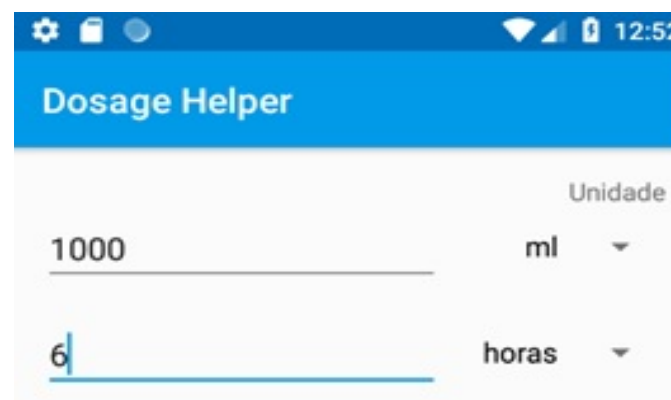

\section{Resultado: 56 gotas por minuto}

\section{RESULTADO}

Figura 5 - Cálculo de gotejamento

Figura 6 - Resultado da simulação de gotejamento

A sétima tela (Figura 7), contém as instruções para o manuseio do aplicativo.

A avaliação do aplicativo foi realizada por duas docentes do Curso de Graduação de Enfermagem, demonstrando para elas, o processo do cálculo de diluição de medicamentos, bem como do cálculo de gotejamento de soro, pontuando como realizar corretamente o preenchimento das variáveis necessárias para os cálculos.

As docentes manusearam o aplicativo e executaram vários exemplos que costumam ensinar em sala de aula para seus alunos. Em relação a esse contexto houveram diversas observações positivas e as docentes não tiveram problemas no manuseio do 
aplicativo. Durante a avaliação puderam escolher em qual unidade desejavam a resposta, indicaram os dados para o cálculo e visualizaram o resultado em uma nova interface, que possui um texto descrevendo o cálculo realizado e o resultado. Esses serão os passos que os alunos deverão seguir para utilizar o OA em seu processo ensino-aprendizagem.
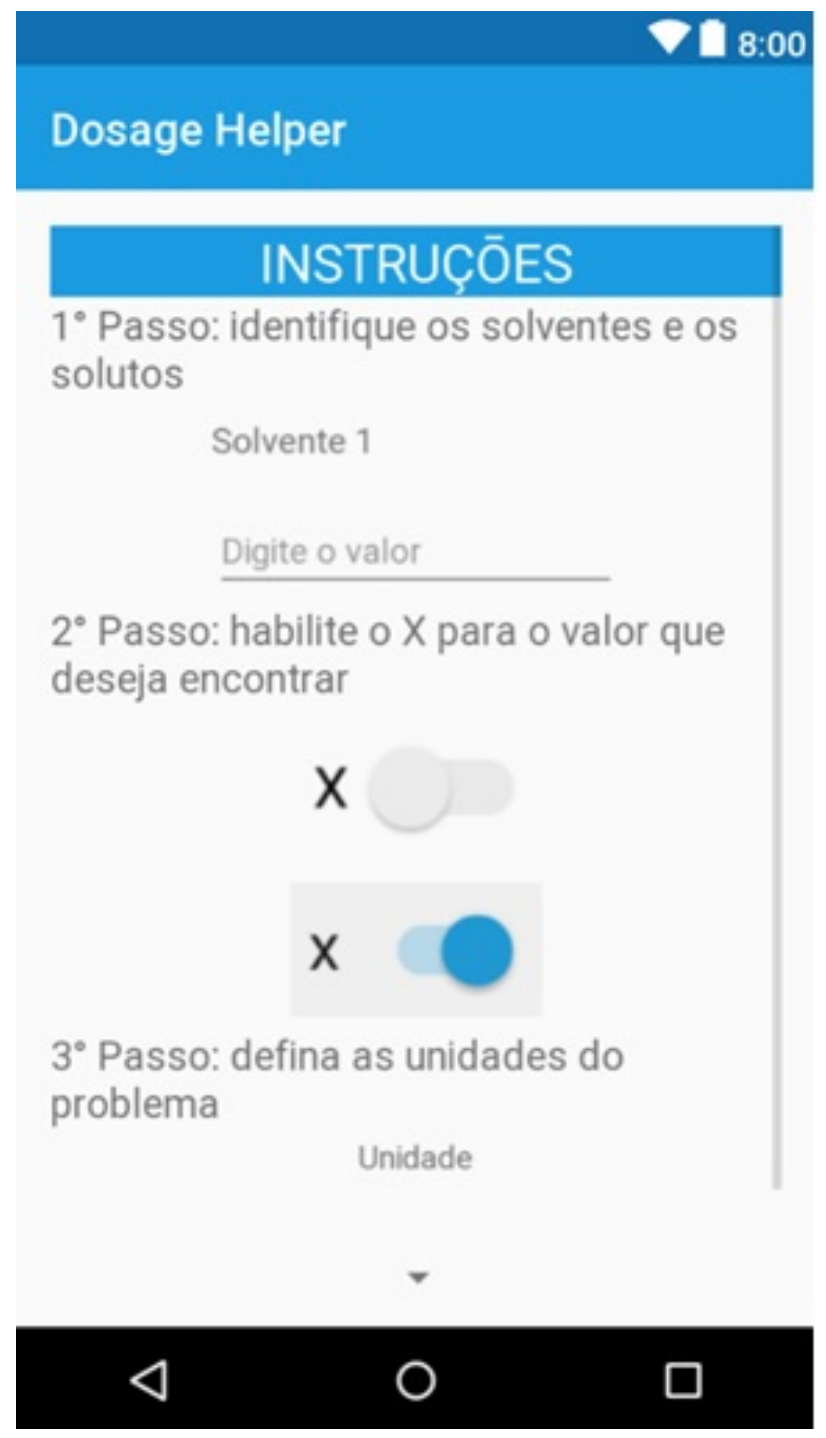

Figura 7 - Instruções para uso do aplicativo

As docentes relataram que os estudantes têm dificuldade com as unidades de medida e cálculo de diluição de medicamentos, conhecimentos necessários para a resolução dos exercícios que são propostos em sala de sala, como por exemplo, o problema apresentado na Figura 8. Também ressaltaram que o Dosage Helper será uma ferramenta relevante para auxiliar os estudantes no processo de ensino-aprendizagem desse contexto. 


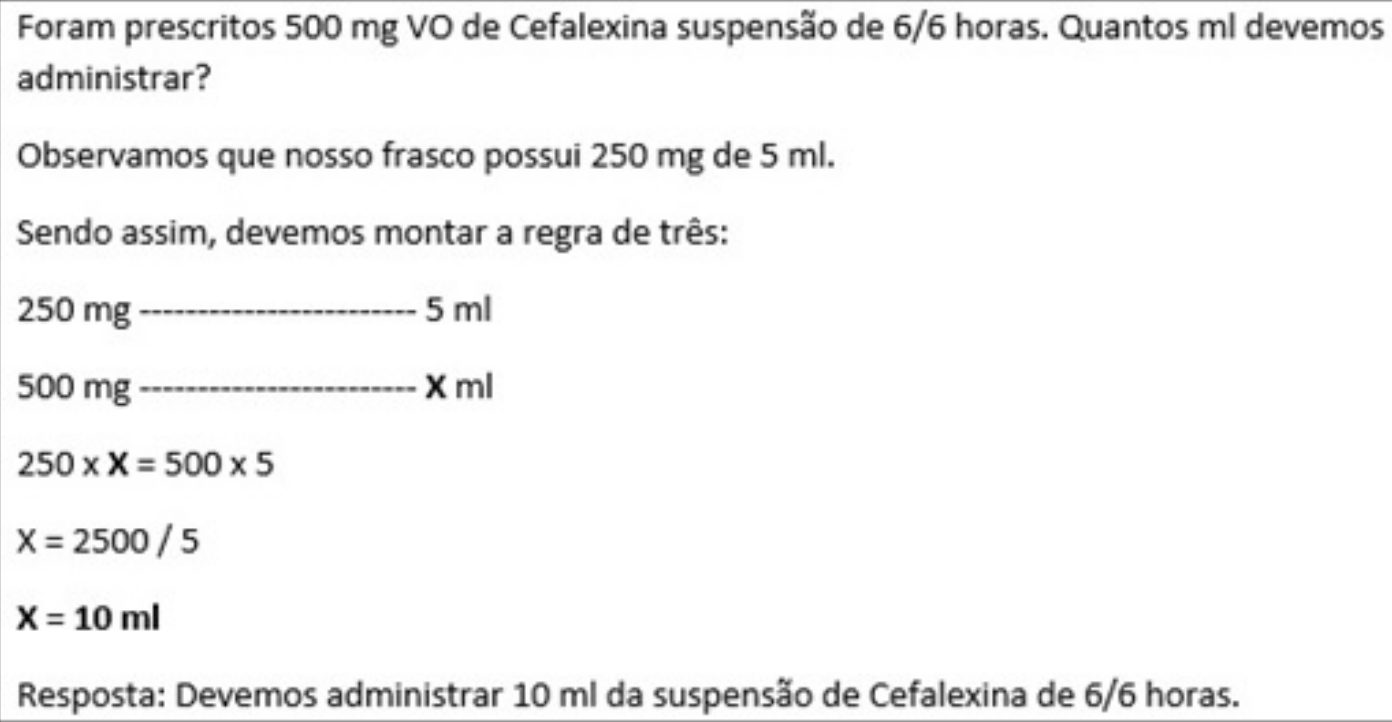

Figura 8 - Exemplo utilizado pelas docentes para validação do OA

As docentes ainda referiram que as interfaces são amigáveis, o tempo de retorno das informações é adequado e os cálculos de diluição de medicamentos e gotejamento de soro são realizados corretamente, portanto, não há necessidade de modificações no app. Desse modo, poderá ser disponibilizado para uso dos alunos de enfermagem de uma Instituição de Ensino Superior como recurso didático-pedagógico para o apoio ao processo de ensino-aprendizagem.

$\mathrm{Na}$ avaliação realizada pelas docentes não houveram manifestações negativas sobre 0 OA desenvolvido, sendo que reiteraram a importância do recurso que o app possui de retornar à forma como o aluno montou a operação, permitindo evidenciar onde ocorreu $\mathrm{o}$ erro, por ocasião da realização do cálculo.

\section{CONCLUSÃO}

O OA engloba conteúdos referentes ao cálculo e diluição de medicamentos, além do cálculo de gotejamento de soro, apoiando o aluno no processo ensino-aprendizagem relativo ao contexto estudado, pois a partir do app o aluno exercita a seleção dos valores indicados nas prescrições médicas e estimula seu raciocínio lógico para a tomada de conduta em relação a preparação de medicação, e como haverá estímulos cognitivos, é possível inferir que o OA auxiliará o educando a aprender a aprender.

O aplicativo poderá ser oferecido como ferramenta de apoio nas aulas de cursos presenciais, na modalidade de Educação a Distância (EAD) ou híbrido (semipresenciais), pois permite aos alunos aplicarem a teoria, além de simular a prática, 
favorecendo o aprender a saber-fazer, exercitando assim, as suas habilidades com vistas a alcançar competências relativas ao cálculo e diluição de medicamentos, bem como gotejamento de soro.

Portanto, ressalta-se que o app será mais uma estratégia didático-pedagógica inerente aos anseios das gerações contemporâneas que ocupam os espaços das escolas de educação superior, uma vez que as estimulam na continuidade da simulação do processo de cálculo de gotejamento de soro, e cálculo e diluição de medicamentos, intra e extra sala de aula.

\section{REFERÊNCIAS BIBLIOGRÁFICAS}

BATES, D. H. et al. Patient risk factors for adverse drug events in hospitalized patients. Archives of Internal Medicine. London, v. 159, n. 21, p. 2553-2560, 1999.

BISTANE, R. H.; CARITÁ, E. C.; SILVA, S. S.; VITTI, S. V. Objetos Virtuais de Aprendizagem para Área da Saúde: uma revisão de literatura. XIV Congresso Brasileiro de Informática em Saúde - CBIS'14, Santos/SP, 07 a 10 de dezembro de 2014.

BLANC, S.; BENLLOCH-DUALDE, J. V. Digital Learning Object Production in Engineering Courses. IEEE Revista Iberoamericana de Tecnologias del Aprendizaje. Espanha, v. 9, n. 2, p. 43-48, 2014.

CARVALHO, V. T. Erros na administração de medicamentos: análise de relatos dos profissionais de enfermagem. 2000. 139f. Dissertação (Mestrado em Enfermagem Fundamental), Escola de Enfermagem de Ribeirão Preto/USP, Universidade de São Paulo, Ribeirão Preto, 2000.

CONSELHO REGIONAL DE ENFERMAGEM - COREn. Boas Práticas - cálculo seguro. v. 1. Cálculo e diluição de medicamentos. São Paulo: COREn, 2011.

GASKELL, G. Entrevistas individuais e grupais. In: GASKELL, G.; BAUER, M. W. (Org.). Pesquisa qualitativa com texto, imagem e som: um manual prático. Petrópolis: Vozes, 2002. p. 64-89.

KITZINGER, J. Focus groups with users and providers of health care. In: POPE, C.; MAYS, N. (Org.). Qualitative research in health care. $2^{\underline{a}}$ ed. London: BMJ Books, 2000 
MCGREAL, R. Learning objects: a practical definition. 2004. Disponível em: . Acessado em 25/04/2018. 